\title{
Effects of a multi-component nutritional telemonitoring intervention on nutritional status, diet quality, physical functioning and quality of life of community-dwelling older adults
}

\author{
Marije N. van Doorn-van Atten ${ }^{1 *}$, Annemien Haveman-Nies ${ }^{1,2}$, Marit M. van Bakel $^{3}$, Monique Ferry ${ }^{3}$, \\ Maite Franco $^{4}$, Lisette C. P. G. M. de Groot ${ }^{1}$ and Jeanne H. M. de Vries ${ }^{1}$ \\ ${ }^{1}$ Division of Human Nutrition, Wageningen University \& Research, PO Box 17, 6700 AA Wageningen, The Netherlands \\ ${ }^{2}$ Strategic Communication Chair, Wageningen University \& Research, P.O. Box 17, 6700 AA Wageningen, The Netherlands \\ ${ }^{3}$ Unité de Recherche en Epidémiologie Nutritionnelle, Université Paris 13, 75 rue Marcel Cachin, F-93017 \\ Bobigny cedex, France \\ ${ }^{4}$ Consorci Sanitari de Terrassa, Ctra Torrebonica s/n, E-08227 Terrassa, Spain
}

(Submitted 6 December 2017 - Final revision received 25 January 2018 - Accepted 6 March 2018)

\section{Abstract}

This study aimed to evaluate the effects of an intervention including nutritional telemonitoring, nutrition education, and follow-up by a nurse on nutritional status, diet quality, appetite, physical functioning and quality of life of Dutch community-dwelling elderly. We used a parallel arm pre-test post-test design with 214 older adults (average age 80 years) who were allocated to the intervention group ( $n$ 97) or control group ( $n$ 107), based on the municipality. The intervention group received a 6-month intervention including telemonitoring measurements, nutrition education and follow-up by a nurse. Effect measurements took place at baseline, after 4.5 months, and at the end of the study. The intervention improved nutritional status of participants at risk of undernutrition $(\beta$ (T1) $=2 \cdot 55 ; 95 \%$ CI $1 \cdot 41,3 \cdot 68 ; \beta$ (T2) $=1 \cdot 77 ; 95 \%$ CI $0 \cdot 60$, $2 \cdot 94$ ) and scores for compliance with Dutch guidelines for the intake of vegetables ( $\beta=1 \cdot 27 ; 95 \%$ CI $0 \cdot 49,2 \cdot 05)$, fruit $(\beta=1 \cdot 24 ; 95 \%$ CI $0 \cdot 60$, $1 \cdot 88)$, dietary fibre $(\beta=1 \cdot 13 ; 95 \%$ CI $0 \cdot 70,1 \cdot 57)$, protein $(\beta=1 \cdot 20 ; 95 \%$ CI $0 \cdot 15,2 \cdot 24)$ and physical activity $(\beta=2 \cdot 13 ; 95 \%$ CI $0 \cdot 98,3 \cdot 29)$. The intervention did not have an effect on body weight, appetite, physical functioning and quality of life. In conclusion, this intervention leads to improved nutritional status in older adults at risk of undernutrition, and to improved diet quality and physical activity levels of communitydwelling elderly. Future studies with a longer duration should focus on older adults at higher risk of undernutrition than this study population to investigate whether the impact of the intervention on nutritional and functional outcomes can be improved.

\section{Key words: Older adults: Undernutrition: eHealth: Lifestyle intervention: Effect evaluation}

Undernutrition adversely affects older adults' health and quality of life and can be caused by a variety of physiological, pathological, psychological and social factors ${ }^{(1-3)}$. Undernutrition is prevalent across the continuum of care, with the highest prevalence observed in the rehabilitation setting $(50.5 \%)$, followed by the hospital (38.7\%), the nursing home (13.8\%) and the community $(5 \cdot 8 \%)^{(4)}$. In absolute numbers, however, most undernutrition is encountered in the community as the majority of older adults lives independently ${ }^{(5)}$. Treatment of undernutrition with oral nutritional supplements increases body weight in older adults, but functional benefit from supplementation has not yet been assessed ${ }^{(6)}$. As it appears to be difficult to reverse the adverse effects of undernutrition, attention should be paid to the prevention of $\mathrm{it}^{(7)}$.

Undernutrition may be addressed by screening practices and nutrition education. Screening allows a targeted effort of time and resources on individuals at the greatest risk, resulting in a widespread demand for nutritional screening in at-risk populations $^{(8)}$. In the Netherlands, only one-quarter of home care clients is structurally screened for undernutrition and health care professionals (HCP) and older adults seem unaware of the problem $^{(9,10)}$. Awareness of the importance of optimal nutritional status for healthful aging may be addressed by nutrition education or counselling. Moreover, during counselling, HCP identify and address risk factors for malnutrition including health, social, economic and geographical factors ${ }^{(11,12)}$. However, the value of nutrition education for elderly remains under recognised and nutrition education research among older adults is scarce ${ }^{(13,14)}$.

Nutritional screening and nutrition education might be addressed using eHealth, which is defined as health services and information delivered or enhanced through the internet and related technologies ${ }^{,(15)}$. eHealth is expected to contribute to

Abbreviations: DHD-FFQ, Dutch Healthy Diet FFQ; HCP, health care professionals.

* Corresponding author: M. N. van Doorn-van Atten, email Marije.vandoorn@wur.nl 
more efficient ways of providing high-quality health care to an aging population with increased pressure on health care resources $^{(16)}$. To our knowledge, eHealth has not yet been used for nutritional screening and nutrition education in a communitydwelling elderly population. We present an intervention that combines nutritional screening in the form of telemonitoring with computer-tailored nutrition education. The aim of this study was to evaluate the effects of this intervention on the primary outcome nutritional status and secondary outcomes diet quality, appetite, physical functioning and quality of life.

\section{Methods}

\section{Study design}

The study followed a parallel arm pre-test post-test design and took place from April 2016 until June 2017. The intervention had a duration of 6 months, and effect measurements took place during screening, at baseline (T0), after 4.5 months (T1) and at the end of the study (T2). In addition, telemonitoring measurements of nutritional status, appetite and diet quality took place in the intervention group as part of the intervention at the beginning of the study (TM0) and 3 months after the start of the study (TM1). The first measurements of nutritional status, appetite and diet quality were used for both effect evaluation and telemonitoring purposes (T0/TM0) (Fig. 1). The study was registered at ClinicalTrials.gov (identifier NCT03240094), url: http://bit.ly/2zFTs3P.

\section{Ethical approval}

This study was conducted according to the guidelines laid down in the Declaration of Helsinki and all procedures involving participants were approved by the Medical Ethical Committee of Wageningen University, no. NL53619.081.1. Written informed consent was obtained from all participants.

\section{Participants}

Recruitment took place from February 2016 until September 2016. Allocation of participants to the treatment group took place on the level of the municipality. The involved care organisations appointed five municipalities where nurses and dieticians were available to implement the intervention. Four other municipalities were allocated to the control group. As a result, participants in the intervention group were recruited from the municipalities of Ermelo, Harderwijk, Nunspeet, Putten and Renkum in the Netherlands. Participants in the control group were recruited from the municipalities of Ede, Rhenen, Veenendaal and Wageningen in the Netherlands. Participants were recruited via invitation letters from care organisations Zorggroep Noordwest-Veluwe and Opella, via advertisements in local newspapers and public spaces, and via invitation per post. Persons could participate when they were 65 years or older and received home care and/or lived in sheltered accommodation or a service flat. Persons who showed interest to participate were visited by a researcher to receive more information about the study, to ask questions, to sign the informed consent, and to be screened on the exclusion criteria. Individuals were excluded from participation if they were cognitively impaired (Mini-Mental State Examination (MMSE) < 20), had diagnosed cancer, received terminal care, were bedridden or bound to a wheelchair, or were unable to watch television. In total, 215 persons were screened for eligibility, of whom ninetyseven were assigned to the intervention group and 107 to the control group, based on the municipality. In the intervention group, twenty-one participants were lost to follow-up, mainly due to health problems or perceived difficulties with the telemonitoring technology. In the control group, six participants were lost to follow-up due to various reasons (Fig. 2).

\section{Intervention}

The PhysioDom Home Dietary Intake Monitoring (HDIM) intervention consisted of the following components: telemonitoring, nutrition education and follow-up by a nurse. These components are described in more detail below.

Telemonitoring. Participants were asked to perform selfmeasurements of body weight (weekly), steps (1 week/ month), and blood pressure (monthly or bi-monthly, and only for a subsample of participants upon indication of a nurse). For

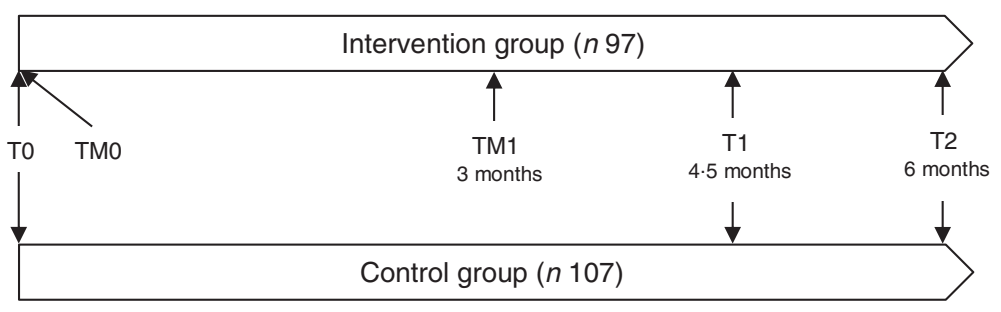

\begin{tabular}{|c|c|c|c|}
\hline Nutritional status & $x$ & $x$ & $\mathrm{x}$ \\
\hline Diet quality & $x$ & $x$ & $\mathrm{x}$ \\
\hline Appetite & $\mathrm{X}$ & $x$ & $\mathrm{x}$ \\
\hline Physical functioning & $x$ & & \\
\hline Quality of life & $x$ & & $x$ \\
\hline
\end{tabular}

Fig. 1. Study design of the PhysioDom Home Dietary Intake Monitoring intervention in the Netherlands including effect measurements (T0, T1 and T2) and telemonitoring measurements (TM0 and TM1). 


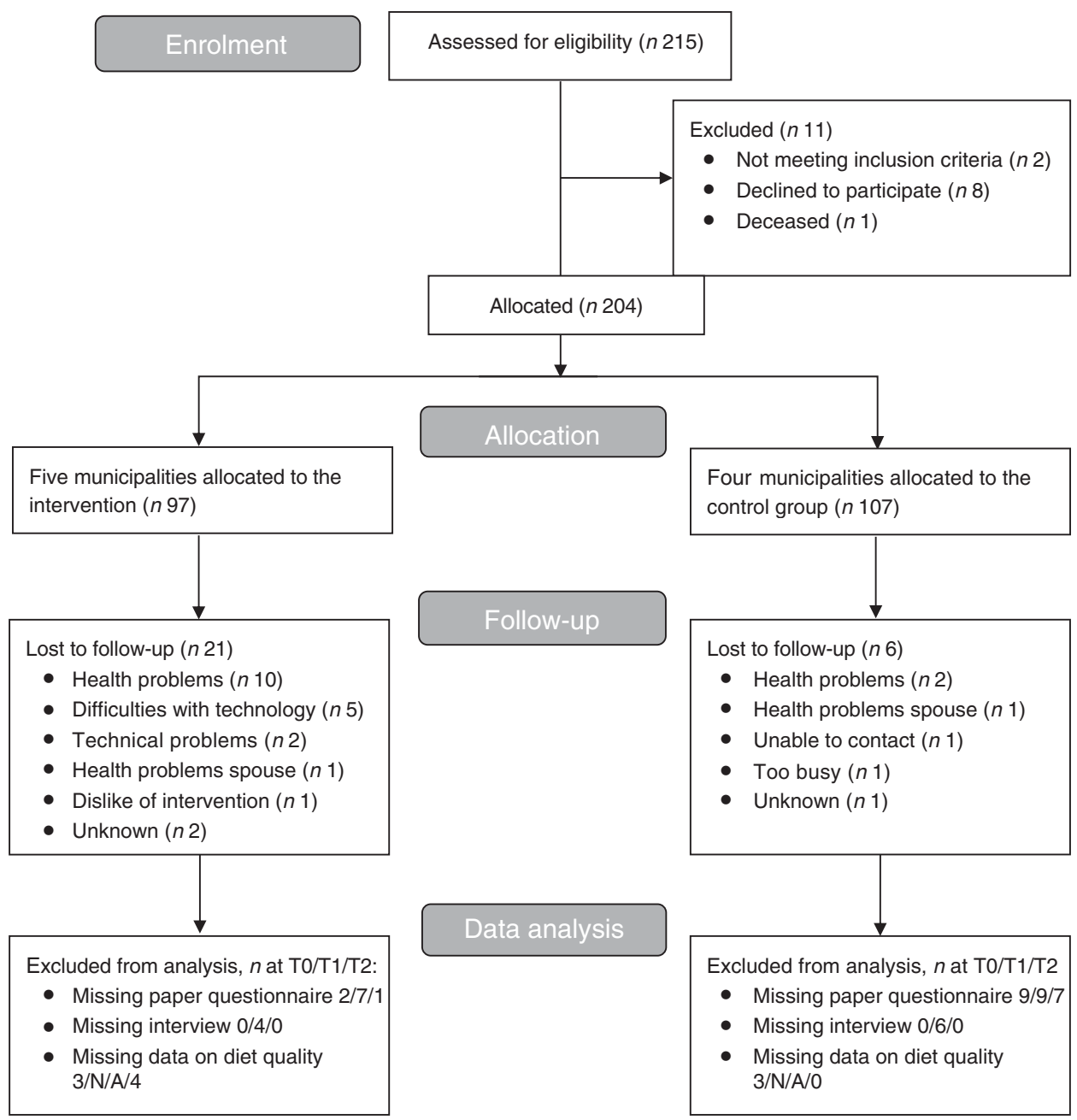

Fig. 2. Flow diagram of participants of the PhysioDom Home Dietary Intake Monitoring intervention in the Netherlands.

these measurements, participants received a weighing scale (type UC-411PBT-C; A\&D), a pedometer (type UW-101; A\&D) and a sphygmomanometer (type UA-767PBT-CI; A\&D), respectively. The weighing scale and sphygmomanometer were connected via Bluetooth to a set-top box. This box was connected to the participant's television. In this way, the telemonitoring results were automatically displayed on the participant's television. Furthermore, telemonitoring results were sent from the set-top box to the nurses via a secured internet connection. Furthermore, participants filled out questionnaires about nutritional status with the Mini Nutritional Assessment Short-Form (MNA-SF) ${ }^{(17)}$, appetite with the Simplified Nutritional Appetite Questionnaire (SNAQ) ${ }^{(18)}$ and diet quality with the Dutch Healthy Diet FFQ (DHD-FFQ) ${ }^{(19)}$. These questionnaires were administered at the start of the study by means of an interview with a researcher (T0/TM0) and 3 months after the start of the study during a telephone interview with a researcher or using their own computer or tablet received from the researchers (TM1) (Fig. 1). The TM1 measurement of some participants was performed during the T1 measurement with researchers due to difficulties with filling out the questionnaires on a computer or tablet. Participants could view the results of these telemonitoring measurements on their television and received feedback on the results.

Nutrition education. Participants received computer-tailored and non-tailored information about nutrition. The computertailored information contained two letters with the results of the DHD-FFQ that were sent after the administration of the DHDFFQ at T0/TM0 and TM1. The results included scores (0-10) for compliance with Dutch guidelines for several nutrients and food groups and physical activity (see Outcomes section) and computer-tailored advice on how to improve compliance. These computer-tailored advice appeared automatically after filling out the DHD-FFQ on a website. Per nutrient or food group, two (for trans-fatty acids) or five (for the other nutrients and food groups) different advice could be given, according to the score for that specific nutrient or food group. For example, participants with low scores for vegetable intake received suggestions that were easily accessible and that should fit with various reasons for not consuming vegetables (e.g. not liking vegetables), whereas participants with high scores for vegetable intake received suggestions that aimed at maintaining this 
behaviour and at having sufficient variation in vegetables. The non-tailored information consisted of three short and general television messages ( $<500$ characters) that were sent weekly to the participants and that targeted determinants of dietary and physical activity behaviour such as awareness, knowledge and attitude. For example, topics included dental health, eating alone $v$. eating with others, and how to enhance the taste of the meal in case of impaired taste perception.

Follow-up by a nurse. In total, seven nurses and three dieticians were involved in the study. Each participant was assigned to a nurse who worked in the same municipality as where the participant lived. Nurses received the telemonitoring results and interpreted these with the help of alerts that were activated in case of undernutrition or the risk of undernutrition, obesity or new blood pressure measurements. Thresholds for activation of alerts are described elsewhere ${ }^{(20)}$. Nurses decided about follow-up of alerts with the help of decision trees ${ }^{(20)}$. In case of risk of undernutrition, the nurse investigated on the causes by looking into the questionnaire results and by contacting the participants to ask more in-depth questions about the personal situation of the participant and possible risk factors. The nurse also advised participants on how to improve protein and energy intake and gave a brochure with advice on this. Studies have indicated the potential of nutrition counselling by a HCP to impact nutritional outcomes in older adults ${ }^{(21-24)}$. In case of undernutrition and obesity, the nurse discussed with the participant whether referral to a general practitioner (GP) or dietitian was desired for professional treatment. In case of undernutrition or risk of undernutrition, the HCP advice had priority over the advice from the DHD-FFQ, although HCP could use the results from the DHD-FFQ to prioritise in the individual advice that was given to participants. In case of abnormal blood pressure measurements, the nurses followed their regular care pathways.

\section{Implementation}

To guide the quality of implementation, the researchers held four preparatory meetings with the involved HCP, held monthly to bi-monthly evaluation meetings with HCP during the study, provided individual at-home training to participants, and provided manuals and a support desk to the HCP and participants. The preparatory meetings for the HCP's lasted one and a half hour each. During these meetings, the HCP received all the information, materials and training needed to provide proper follow-up of telemonitoring measurements to participants. The nurses also received a workshop from a dietitian with the aim to improve knowledge about nutrition and undernutrition in older adults. The individual at-home training for participants took approximately $45 \mathrm{~min}$ and covered the use of the television channel, the weighing scale, the pedometer and, if applicable, the sphygmomanometer and/or tablet. Researchers stimulated compliance with the intervention by giving participants a paper calendar with the telemonitoring measurements, illustrated cards with cues to use the television channel and to perform the telemonitoring measurements, and three newsletters. Participants in the control group received usual care. Domestic care was received by $80 \%$ of the control group participants, personal care and nursing care by $30 \%$ and $3 \%$, respectively.

The intervention including its theoretical framework is described in more detail elsewhere ${ }^{(20)}$.

\section{Outcomes}

Measurements took place during the screening visit, at T0, T1 and $\mathrm{T} 2$. In addition, telemonitoring measurements took place at TM0 and TM1 as part of the telemonitoring intervention in the intervention group only (Fig. 1). Data were collected with paper questionnaires and through structured interviews at the participants' homes performed by trained researchers or research assistants. Baseline characteristics were recorded during the screening visit in the intervention group and at T0 in the control group. Baseline characteristics included age, sex, height, BMI $\left(\mathrm{kg} / \mathrm{m}^{2}\right)$, education level, birth country, marital status, living situation (alone or with partner or relatives) and current diagnoses. These items were derived from The Older Persons and Informal Caregivers Survey Minimum DataSet (TOPICSMDS) ${ }^{(25)}$. Furthermore, cognitive functioning was measured with the $\mathrm{MMSE}^{(26)}$ and dental problems, swallowing problems, type and amount of care or informal care, presence of a diet and wish for weight reduction were recorded. The primary outcome nutritional status was assessed with the Mini Nutritional Assessment at T0/TM0, T1 and $\mathrm{T} 2^{(27)}$. A higher MNA score means a better nutritional status, with a score from 0-16 indicating undernutrition, a score from $17-23.5$ risk of undernutrition, and a score from $24-30$ a normal nutritional status. In addition, nutritional status was assessed at T0/TM0 and TM1 in the intervention group as part of the telemonitoring intervention, using the MNA-SF ${ }^{(17)}$. Body weight was measured at T0, T1 and $\mathrm{T} 2$ by researchers to the nearest $0.1 \mathrm{~kg}$ using a scale of the brand A\&D, type UC-411PBT-C. Participants were asked to take off their shoes and heavy clothes before the measurement. In addition, participants in the intervention group measured their body weight weekly as part of the telemonitoring intervention. Diet quality was measured with the DHD-FFQ at T0/TM0, TM1 and $\mathrm{T} 2{ }^{(19)}$. The DHD-FFQ has twenty-eight items and evaluates the compliance with Dutch dietary guidelines. These guidelines are formulated for the general population of 2 years and older and include vegetables, fruit, fish, alcohol, SFA, trans-fatty acids, $\mathrm{Na}$ and dietary fibre ${ }^{(28)}$. In addition, the DHD-FFQ assesses compliance with Dutch guidelines for physical activity $^{(28)}$. For this study, compliance with guidelines for protein and vitamin $\mathrm{D}$ was also assessed, taking into account that older adults require a higher intake vitamin $\mathrm{D}$ and protein than a younger population ${ }^{(28,29)}$. The intake of these nutrients could be assessed by the DHD-FFQ as this questionnaire includes questions on all relevant protein and vitamin $\mathrm{D}$ rich food groups consumed by an elderly Dutch population ${ }^{(30)}$. Based on the level of compliance with a guideline, a score between 0 and 10 was composed with higher scores indicating better compliance with the guideline. Furthermore, a total score ranging from 0 to 80 indicates overall diet quality and is calculated by summing the scores for vegetables, fruit, fish, alcohol, SFA, trans-fatty acids, $\mathrm{Na}$ and dietary fibre. Appetite was measured with the SNAQ questionnaire at T0/TM0, TM1 and T2 ${ }^{(18)}$. Level of 
independence of activities of daily living and physical functioning were measured at T0 and T2 with the Katz-15 questionnaire and Short Physical Performance Battery, respectively ${ }^{(31,32)}$. Quality of life was measured with the Short Form 36 questionnaire at T0, T1 and $\mathrm{T} 2^{(33,34)}$.

\section{Statistics}

Sample size calculation. We aimed to detect a difference in MNA change of three and assumed a standard deviation of $6 \cdot 1$, based on previous research ${ }^{(35)}$. Furthermore, we took into account a two-sided significance level of 0.05 and a power of $80 \%$. Based on the formula $2 \times \frac{\left[(Z \alpha / 2+Z \beta)^{2} \times \sigma^{2}\right]}{\delta^{2}}$, with $\alpha=0.05$, $\beta=0 \cdot 80, \sigma=6 \cdot 1$ and $\delta=3$, we needed a sample size of sixty-five participants per group. Allowing for a drop-out rate of $30 \%$ at maximum, we needed a sample size of ninety-three participants in each group.

Data were analysed with SPSS version 22. Descriptive data were presented as means and standard deviations or as percentages. Statistical analysis was carried out according to the intention-to-treat principle. Baseline differences between the intervention and control group were analysed with an independent $t$ test or a $\chi^{2}$ test. Differences in changes between the intervention and control group were analysed using linear mixed models. Therefore, we first specified a model as large as possible for the fixed and random part, for example, a saturated model with all main effects and interactions and an unstructured covariance matrix. Then we simplified the covariance model by specifying simpler covariance structures and testing them with (REML) LR test until a model was obtained that was as parsimonious as possible. Finally, we simplified the fixed part of the model by including dummy's for T1, T2, treatment group, the interaction terms of the dummy's for T1 and T2 and treatment group, age, sex and if necessary also other covariates that influenced the effect estimates. The analysis of the primary outcome nutritional status also included investigation of a possible interaction of the intervention with baseline nutritional status, categorised into normal nutritional status (MNA $\geq 24$ ) or having undernutrition or risk of undernutrition (MNA $\leq 23 \cdot 5$ ). Furthermore, for the study outcome body weight, we investigated a possible interaction of the intervention with desire to lose weight as about half of the participants in the intervention group desired to lose weight. Finally, we used logistic regression to analyse the effects of the intervention on the score for compliance with the dietary guideline for trans-fatty acids, as this score could be either 0 or 10 .

\section{Results}

Table 1 shows the baseline characteristics of the study population. Participants in the intervention group were slightly younger and had a higher BMI than participants in the control group. Furthermore, participants in the intervention group lived less often alone, were less often on a diet, and received more often informal care than participants in the control group.

Participants who dropped out of the study were significantly older and had a lower MMSE score. They were also significantly more likely to have swallowing problems and to receive personal care and/or nursing care at home. Furthermore, participants who dropped out had a worse physical functioning and were less physically active than participants who completed the study.

Table 2 shows the crude means of the study outcomes. At T0, participants in the intervention group had a significantly lower compliance with guidelines for the intake of vegetables and fibre $(t(197)=2 \cdot 15, P=0 \cdot 03), t(197)=2 \cdot 64, P=0.009$, respectively $)$, and a lower compliance with guidelines for physical activity $((t)(197)=3 \cdot 31, P=0 \cdot 001)$, than participants in the control group. The intervention group had significantly better compliance with guidelines for intake of trans-fatty acids than the control group at T0 $\left(\chi^{2}(1, n\right.$ 199) $=4.63, P=0.03)$.

Participants in the intervention and control group did not significantly differ in changes over time in the primary outcome nutritional status. However, we observed a significant interaction of the intervention with baseline nutritional status. Intervention group participants with a poor nutritional status at baseline improved significantly more in MNA score than control group participants with a poor nutritional status at baseline. Participants in the intervention and control group with a normal nutritional status at baseline did not significantly differ in changes over time in MNA score $(\beta(\mathrm{T} 1)=2.55 ; 95 \%$ CI 1.41 , 3.68; $\beta$ (T2) 1.77 ; $95 \%$ CI 0.60, 2.94, Table 2). Furthermore, we did not find a significant effect of the intervention on body weight. Intervention group participants without a desire to lose weight increased more in body weight than control group participants without a desire to lose weight, although this was not statistically significant (Table 2).

The intervention did not have an effect on the total score for diet quality, but participants in the intervention group significantly increased their compliance with several Dutch dietary guidelines, compared to the control group. They significantly increased their compliance with the guidelines for vegetables ( $\beta=1.27 ; 95 \%$ CI $0.49,2 \cdot 05)$, fruit $(\beta=1.24 ; 95 \%$ CI $0.60,1 \cdot 88)$, dietary fibre $(\beta=1.13 ; 95 \%$ CI $0.70,1.57)$ and protein $(\beta=1.20$; $95 \%$ CI $0 \cdot 15,2 \cdot 24)$. Furthermore, participants in the intervention group slightly decreased their compliance with the guideline for the intake of $\mathrm{Na}$, whereas participants in the control group increased their compliance with this guideline. This difference in change over time was significant $(\beta=-0.97$; $95 \% \mathrm{CI}-1 \cdot 77$, $-0 \cdot 17)$. The intervention did not have an effect on the remaining Dutch dietary guidelines for the intake of fish, SFA, trans-fatty acids, alcohol and vitamin D. Participants in the intervention group significantly improved their compliance with the Dutch guidelines for physical activity, compared to the control group $(\beta=2 \cdot 13 ; 95 \%$ CI $0 \cdot 98,3 \cdot 29)$.

Finally, participants in the intervention and control group did not significantly differ in changes over time in appetite, physical functioning and quality of life (Table 2).

\section{Discussion}

The aim of this study was to evaluate the effects of the PhysioDom HDIM intervention on nutritional status, diet quality, appetite, physical functioning and quality of life. The intervention was effective in improving nutritional status in participants at risk of undernutrition and in improving several items of 
Table 1. Baseline characteristics of participants of the PhysioDom Home Dietary Intake Monitoring study (Mean values and standard deviations; percentages)

\begin{tabular}{|c|c|c|c|c|c|}
\hline & \multicolumn{2}{|c|}{ Intervention group ( $n$ 97) } & \multicolumn{2}{|c|}{ Control group ( $n$ 107) } & \multirow[b]{2}{*}{$P^{*}$} \\
\hline & Mean & SD & Mean & SD & \\
\hline Age (years) & 78.4 & $7 \cdot 2$ & $81 \cdot 0$ & 7.9 & 0.02 \\
\hline $\mathrm{BMI}\left(\mathrm{kg} / \mathrm{m}^{2}\right)$ & $29 \cdot 2$ & 4.5 & $27 \cdot 7$ & $5 \cdot 4$ & 0.04 \\
\hline Number of diagnoses & 1.5 & 1.5 & 1.3 & 1.3 & 0.26 \\
\hline MMSE score & $28 \cdot 6$ & 1.5 & $25 \cdot 8$ & 1.9 & 0.69 \\
\hline Sex (\% male $)$ & \multicolumn{2}{|c|}{34} & \multicolumn{2}{|c|}{$23 \cdot 4$} & 0.09 \\
\hline Education level (\%)† & & & & & 0.08 \\
\hline Low & \multicolumn{2}{|c|}{$17 \cdot 5$} & \multicolumn{2}{|c|}{$10 \cdot 3$} & \\
\hline Moderate & \multicolumn{2}{|c|}{$55 \cdot 7$} & \multicolumn{2}{|c|}{49.5} & \\
\hline High & \multicolumn{2}{|c|}{$26 \cdot 8$} & \multicolumn{2}{|c|}{$40 \cdot 2$} & \\
\hline Civil status (\%) & & & & & 0.11 \\
\hline Married & \multicolumn{2}{|c|}{$42 \cdot 3$} & \multicolumn{2}{|c|}{$27 \cdot 1$} & \\
\hline Single & \multicolumn{2}{|c|}{$7 \cdot 2$} & \multicolumn{2}{|c|}{$13 \cdot 1$} & \\
\hline Divorced & \multicolumn{2}{|c|}{$7 \cdot 2$} & \multicolumn{2}{|c|}{$10 \cdot 3$} & \\
\hline Widowed & \multicolumn{2}{|c|}{$43 \cdot 3$} & \multicolumn{2}{|c|}{$49 \cdot 5$} & \\
\hline Living alone (\%) & \multicolumn{2}{|c|}{$55 \cdot 7$} & \multicolumn{2}{|c|}{$74 \cdot 8$} & 0.004 \\
\hline Born in the Netherlands (\%) & \multicolumn{2}{|c|}{$96 \cdot 9$} & \multicolumn{2}{|c|}{$90 \cdot 7$} & 0.07 \\
\hline Dental problems (\%) & \multicolumn{2}{|c|}{$18 \cdot 6$} & \multicolumn{2}{|c|}{$15 \cdot 0$} & 0.49 \\
\hline Swallowing problems (\%) & \multicolumn{2}{|c|}{$17 \cdot 5$} & \multicolumn{2}{|c|}{$13 \cdot 1$} & 0.38 \\
\hline Desire to lose weight (\%) & \multicolumn{2}{|c|}{$52 \cdot 7$} & \multicolumn{2}{|c|}{39.4} & 0.07 \\
\hline Currently on a diet (\%) & \multicolumn{2}{|c|}{$9 \cdot 7$} & & & 0.01 \\
\hline Nutritional status (\%) & & & & & 0.45 \\
\hline Normal nutritional status & & & & & \\
\hline At risk of undernutrition & & & & & \\
\hline Undernourished & & & & & \\
\hline Type of care (\%) & & & & & \\
\hline Domestic care & & & & & 0.72 \\
\hline Personal care & & & & & 0.75 \\
\hline Nursing care & & & & & 0.05 \\
\hline Individual support & & & & & 0.27 \\
\hline Informal care & & & & & $<0.001$ \\
\hline Service flat or sheltered housing & & & & & 0.12 \\
\hline
\end{tabular}

diet quality and compliance with guidelines for physical activity. The intervention did not have an effect on body weight, appetite, physical functioning and quality of life.

The intervention led to an improved nutritional status in participants at risk of undernutrition. These participants received a more intense intervention than participants who were not at risk, as they received additional advice by a nurse and a brochure with advice on how to improve protein and energy intake. If necessary, they were referred to their GP or a dietitian. To our knowledge, this is one of the first studies that used eHealth for nutrition screening and nutrition education to improve nutritional status in community-dwelling elderly. Other similar non-eHealth studies consisted of nutrition screening with a validated screening tool, followed by a nutrition intervention such as printed nutrition education material ${ }^{(36)}$, nutrition newsletters and dietary consultation ${ }^{(37)}$, personalised evaluation and consultation ${ }^{(38,39)}$, or a combination of counselling, nutrition education and meals on wheels ${ }^{(40,41)}$. These studies found similar positive effects on the nutritional status of older adults at risk of undernutrition, although many of these studies lack the presence of a control group or rely on self-report ${ }^{(42)}$. Our study adds to these findings by suggesting that eHealth can be used for nutrition screening, but that additional consultation of a HCP remains necessary to achieve an effect on nutritional status.

The intervention group improved compliance with guidelines for the intake of fruit, vegetables, dietary fibre and protein ${ }^{(28,29)}$. An optimal diet quality is essential for older adults as a more nutrient-dense diet is required considering the declined energy requirements and food intake that often accompany aging. Only one other pilot study was found that used eHealth to provide computer-tailored dietary advice to older adults, as part of a web-based platform concerning healthy eating, physical activity, and meaningful social roles. This 8-week intervention appeared to be feasible to implement, but it did not lead to significant effects on dietary intake and physical activity ${ }^{(43)}$. A review of non-eHealth studies that focus on dietary advice and nutrition counselling for older adults reported positive effects on dietary intake ${ }^{(24)}$. For example, a 6-month homebased nutrition education intervention consisting of eight home visits, bi-weekly phone calls and monthly letters resulted in increased fruits and vegetables intakes ${ }^{(44)}$. The review further concludes that comprehensive interventions involving active participation and collaborative elements such as group classes and follow-up meetings are most promising in affecting nutritional outcomes, in contrast to interventions with limited 
Table 2. Study outcomes on T0, T1 and T2, and the interaction terms treatment and time points $\mathrm{T} 1$ and $\mathrm{T} 2$ (Crude means and standard deviations; $\beta$-coefficients and odds ratios and $95 \%$ confidence intervals) $t$

\begin{tabular}{|c|c|c|c|c|c|c|c|c|c|c|c|c|c|c|c|c|c|}
\hline & \multicolumn{6}{|c|}{ Intervention group } & \multicolumn{6}{|c|}{ Control group } & & & & & \\
\hline & \multicolumn{2}{|c|}{ TO/TMO } & \multicolumn{2}{|c|}{ T1/TM1 } & \multicolumn{2}{|c|}{$\mathrm{T} 2$} & \multicolumn{2}{|c|}{ T0 } & \multicolumn{2}{|c|}{ T1 } & \multicolumn{2}{|c|}{$\mathrm{T} 2$} & \multicolumn{5}{|c|}{ Linear mixed models } \\
\hline & Mean & SD & Mean & SD & Mean & SD & Mean & SD & Mean & SD & Mean & SD & $\beta(\mathrm{T} 1)$ & $95 \% \mathrm{Cl}$ & $\beta(\mathrm{T} 2)$ & $95 \% \mathrm{Cl}$ & $n$ \\
\hline \multicolumn{18}{|l|}{ Nutritional status } \\
\hline MNA score $(0-30) \ddagger$ & $25 \cdot 9$ & $2 \cdot 6$ & $26 \cdot 2$ & $2 \cdot 6$ & $26 \cdot 1$ & 2.6 & $26 \cdot 0$ & $2 \cdot 4$ & $25 \cdot 8$ & 2.5 & $25 \cdot 8$ & 2.6 & 0.62 & $-0.07,1.32$ & 0.22 & $-0.48,0.92$ & 188 \\
\hline Normal & $26 \cdot 9$ & 1.7 & $27 \cdot 0$ & $2 \cdot 0$ & $26 \cdot 7$ & 2.6 & $26 \cdot 8$ & 1.6 & $26 \cdot 3$ & $2 \cdot 4$ & $26 \cdot 4$ & $2 \cdot 1$ & 0.09 & $-0.62,0.81$ & -0.17 & $-0.90,0.56$ & 153 \\
\hline At risk & $22 \cdot 2$ & 1.9 & $24 \cdot 7$ & $2 \cdot 0$ & $24 \cdot 2$ & $2 \cdot 3$ & 21.9 & 1.5 & 23.4 & $2 \cdot 6$ & 22.5 & $3 \cdot 0$ & $2 \cdot 55^{\star \star \star}$ & $1.41,3.68$ & $1 \cdot 77^{\star \star}$ & $0.60,2.94$ & 35 \\
\hline Body weight $(\mathrm{kg}) \S$ & $80 \cdot 1$ & 14.0 & $79 \cdot 7$ & 13.6 & $80 \cdot 2$ & $13 \cdot 2$ & 74.0 & 16.5 & $74 \cdot 2$ & 16.0 & 73.9 & $16 \cdot 0$ & 0.30 & $-0.37,0.98$ & 0.32 & $-0.54,1.17$ & 187 \\
\hline No desire for weight loss & $72 \cdot 9$ & $12 \cdot 0$ & 73.5 & $12 \cdot 7$ & 74.8 & 12.9 & $68 \cdot 0$ & $12 \cdot 8$ & $68 \cdot 7$ & $12 \cdot 7$ & 67.9 & $12 \cdot 8$ & 0.72 & $-0.13,1.57$ & 0.97 & $-0.11,2.04$ & 101 \\
\hline Desire for weight loss & 86.0 & $12 \cdot 9$ & $85 \cdot 3$ & $12 \cdot 1$ & 84.9 & 11.9 & $84 \cdot 4$ & $16 \cdot 5$ & 83.4 & $15 \cdot 6$ & 83.8 & $15 \cdot 2$ & -0.06 & $-0.87,0.76$ & -0.38 & $-0.42,0.65$ & 86 \\
\hline Diet quality (DHD score) & & & & & & & & & & & & & & & & & 203 \\
\hline Total score $(0-80)$ & $57 \cdot 3$ & $10 \cdot 0$ & $60 \cdot 8$ & 9.4 & $59 \cdot 8$ & $10 \cdot 4$ & $56 \cdot 5$ & $10 \cdot 2$ & $\mathrm{~N} / \mathrm{A}$ & & $57 \cdot 1$ & 9.9 & & $\mathrm{~N} / \mathrm{A}$ & 1.42 & $-1 \cdot 42,4 \cdot 26$ & \\
\hline \multicolumn{18}{|l|}{ Sub scores $(0-10)$} \\
\hline Vegetables & $6 \cdot 3$ & 2.7 & $7 \cdot 0$ & $2 \cdot 7$ & 7.9 & $2 \cdot 6$ & $7 \cdot 2$ & $2 \cdot 7$ & $\mathrm{~N} / \mathrm{A}$ & & $7 \cdot 2$ & 2.9 & & $\mathrm{~N} / \mathrm{A}$ & $1 \cdot 27^{*}$ & $0.49,2.05$ & \\
\hline Fruits & $8 \cdot 2$ & $2 \cdot 4$ & $9 \cdot 1$ & 1.9 & $9 \cdot 1$ & $2 \cdot 0$ & 8.7 & 2.5 & $\mathrm{~N} / \mathrm{A}$ & & $8 \cdot 4$ & 2.9 & & $\mathrm{~N} / \mathrm{A}$ & $1 \cdot 24^{\star \star \star}$ & $0.60,1.88$ & \\
\hline Dietary fibres & $7 \cdot 4$ & 1.8 & $8 \cdot 2$ & 1.7 & 8.4 & 1.5 & $8 \cdot 1$ & 1.8 & $\mathrm{~N} / \mathrm{A}$ & & 7.9 & $2 \cdot 1$ & & $\mathrm{~N} / \mathrm{A}$ & $1 \cdot 13^{\star \star \star}$ & $0.70,1.57$ & \\
\hline Fish & 5.4 & 3.2 & $6 \cdot 2$ & 3.3 & 5.4 & 3.0 & 5.3 & 3.4 & $N / A$ & & $5 \cdot 2$ & 3.7 & & $N / A$ & -0.13 & $-0.98,0.72$ & \\
\hline SFA & $5 \cdot 0$ & 4.3 & $5 \cdot 0$ & $4 \cdot 1$ & 4.5 & $4 \cdot 1$ & 4.5 & 4.0 & $\mathrm{~N} / \mathrm{A}$ & & 4.5 & 4.0 & & $\mathrm{~N} / \mathrm{A}$ & -0.54 & $-1.72,0.64$ & \\
\hline Trans-fatty acids (\% compliant II) & 83.2 & & 88.6 & & $80 \cdot 8$ & & $70 \cdot 2$ & & $N / A$ & & 73.3 & & & $\mathrm{~N} / \mathrm{A}$ & OR 0.77 & $0.35,1.72$ & \\
\hline $\mathrm{Na}$ & 7.0 & 2.6 & 6.9 & $2 \cdot 9$ & 6.6 & $2 \cdot 9$ & $6 \cdot 3$ & $2 \cdot 7$ & $\mathrm{~N} / \mathrm{A}$ & & 6.9 & 2.5 & & $N / A$ & $-0.97^{\star}$ & $-1.77,-0.17$ & \\
\hline Alcohol & 9.5 & 1.4 & 9.5 & 1.6 & $9 \cdot 8$ & 0.9 & $9 \cdot 3$ & $2 \cdot 1$ & $\mathrm{~N} / \mathrm{A}$ & & 9.5 & 1.7 & & $\mathrm{~N} / \mathrm{A}$ & 0.09 & $-0.30,0.48$ & \\
\hline \multicolumn{18}{|l|}{ Extra scores $(0-10)$} \\
\hline Protein & 4.9 & 3.6 & $5 \cdot 7$ & 3.5 & 6.0 & 3.6 & $5 \cdot 3$ & 3.6 & $N / A$ & & $5 \cdot 1$ & 3.8 & & $N / A$ & $1 \cdot 20^{*}$ & $0 \cdot 15,2 \cdot 24$ & \\
\hline Vitamin D & 2.5 & 1.5 & $2 \cdot 9$ & 1.6 & $2 \cdot 7$ & 1.7 & $2 \cdot 2$ & $1 \cdot 1$ & $N / A$ & & $2 \cdot 2$ & 1.3 & & $\mathrm{~N} / \mathrm{A}$ & 0.20 & $-0.11,0.52$ & \\
\hline Physical activity & $5 \cdot 0$ & 4.1 & $6 \cdot 2$ & 4.0 & $6 \cdot 3$ & 3.8 & 6.9 & 3.8 & N/A & & $6 \cdot 0$ & 4.2 & & $\mathrm{~N} / \mathrm{A}$ & $2 \cdot 13^{\star \star \star}$ & $0.98,3.29$ & \\
\hline \multicolumn{18}{|l|}{ Appetite } \\
\hline SNAQ score $(0-20)$ & $15 \cdot 6$ & 1.8 & $15 \cdot 4$ & 1.8 & 15.5 & 1.9 & $15 \cdot 6$ & 1.7 & $\mathrm{~N} / \mathrm{A}$ & & $15 \cdot 6$ & 1.9 & & $\mathrm{~N} / \mathrm{A}$ & -0.19 & $-0.65,0.27$ & 204 \\
\hline \multicolumn{18}{|l|}{ Physical functioning } \\
\hline Katz-15 score $(0-15)$ & $2 \cdot 1$ & 2.7 & $\mathrm{~N} / \mathrm{A}$ & & $2 \cdot 1$ & $2 \cdot 8$ & $2 \cdot 0$ & $2 \cdot 7$ & $N / A$ & & $2 \cdot 0$ & 2.7 & & $\mathrm{~N} / \mathrm{A}$ & 0.17 & $-0.30,0.64$ & 199 \\
\hline SPPB score $(0-12)$ & $7 \cdot 2$ & 3.1 & $\mathrm{~N} / \mathrm{A}$ & & $7 \cdot 2$ & 2.9 & $7 \cdot 2$ & 3.3 & $\mathrm{~N} / \mathrm{A}$ & & $6 \cdot 6$ & 3.5 & & $\mathrm{~N} / \mathrm{A}$ & 0.21 & $-0.32,0.74$ & 203 \\
\hline \multicolumn{18}{|l|}{ Quality of life } \\
\hline SF36 MCS & $46 \cdot 8$ & 9.7 & $47 \cdot 8$ & $10 \cdot 7$ & $47 \cdot 1$ & $10 \cdot 9$ & $49 \cdot 3$ & $9 \cdot 7$ & $48 \cdot 8$ & 9.8 & 49 & $10 \cdot 3$ & $1 \cdot 12$ & $-1.08,3.32$ & 0.76 & $-1.42,2.94$ & 199 \\
\hline SF36 PCS & $37 \cdot 2$ & $10 \cdot 9$ & 38 & $10 \cdot 7$ & 38.1 & $11 \cdot 0$ & 39.8 & $10 \cdot 3$ & 39.8 & $10 \cdot 8$ & 39.8 & $11 \cdot 1$ & -0.44 & $-2 \cdot 08,1.20$ & -0.44 & $-2.06,1 \cdot 18$ & 199 \\
\hline
\end{tabular}

MNA, Mini Nutritional Assessment; DHD, Dutch Healthy Diet; SNAQ, Simplified Nutritional Appetite Questionnaire; SPPB, Short Physical Performance Battery; SF36, Short Form 36; MCS, Mental Component Score; PCS, Physical Component Score.

< $0.01,{ }^{* * *} P<0.001$

† All results are adjusted for age and sex.

f Adjusted for age, sex, swallowing problems, dental problems, cognitive functioning and desire to lose weight.

$\S$ Adjusted for age, sex, and desire to lose weight.

II Analysed using logistic regression. Adjusted for age, sex, and baseline compliance with guidelines for intake of trans-fatty acids. 
personal contact with study participants ${ }^{(24)}$. These type of interventions are costly to implement and expensive to scale up, however, as they require a considerable amount of human resources. Contrary to what this review concluded, our intervention with limited contact between participants and researchers was successful in improving diet quality. This could be explained by the fact that our intervention included personalised dietary advice, which is suggested to be more effective in achieving behaviour change than non-personalised advice $^{(45)}$. Furthermore, the control group unexpectedly improved compliance with guidelines for the intake of salt. This may be a chance finding, or it may be attributed to the fact that more participants in the control group were on a diet, although adjusting for this in the analyses did not alter the results. To conclude, this study shows that using a personalised eHealth approach for nutrition education can be as effective as a noneHealth approach in improving diet quality, with the additional benefits that eHealth is more scalable and sustainable while keeping costs limited ${ }^{(45)}$.

The intervention group significantly improved compliance with guidelines for physical activity. Participants were asked to wear a pedometer for 1 week/month during the intervention period. In addition, they were encouraged to set goals for their daily number of steps. In studies among younger adults, pedometer use is associated with significant increases in physical activity $^{(46)}$. Studies in older adults also suggest that pedometer use can be effective in increasing daily steps ${ }^{(47,48)}$. Our study confirms that pedometer-based interventions appear to be an easy and cheap way to encourage physical activity in community-dwelling older adults, although more research is needed to establish long-term effects.

The intervention did not have effects on body weight, appetite, physical functioning and quality of life. The lack of effects on these type of outcomes is in line with a review by Van den Berg et al. ${ }^{(49)}$, which suggests that eHealth interventions for older adults show better results for behavioural outcomes than for medical outcomes, quality of life and economic outcomes. The lack of effects in this study could possibly be attributed to several aspects. First, a longer intervention duration, a more intense intervention, and a larger sample size might be needed to establish effects on these long-term outcomes. Second, it is suggested that nutritional interventions that are implemented among a wide range of patients with a smaller risk of undernutrition have not demonstrated clinical benefits ${ }^{(8)}$. It is argued that interventions could better target persons who are at higher risk to become malnourished to be able to intervene in a more targeted and specialised way ${ }^{(8)}$. Indeed, less than one-fifth of our study population risked undernutrition. Future research might target a population at greater risk of undernutrition to demonstrate effectiveness in a more homogenous sample, for example through connecting the intervention to care pathways for frail or hospitalised elderly patients.

To our knowledge, this is the first study that used eHealth for a combination of nutritional screening and education among community-dwelling older adults. A strength of this study was that the intervention was embedded within health care organisations, reflecting a real-life setting and thus improving external validity. Other strengths are the use of a theoretical framework including behaviour change techniques and providing tailored dietary advice, making a sustainable behavioural change more probable ${ }^{(45)}$. Furthermore, the addition of scores for compliance with guidelines for protein and vitamin $\mathrm{D}$ to the DHD-FFQ can be regarded as a strength. These nutrients are of particular interest for older adults with regard to bone health and muscle functioning. Adding these components to the original DHD-FFQ resulted in a more relevant dietary advice for our participants. Limitations of the study include the nonrandomised design. Randomisation was not desirable due to the risk of contamination as nurses delivered a large part of the intervention. This led to baseline differences between the intervention and control group. Although we were able to adjust for many possible confounders, we cannot completely exclude residual confounding. Another limitation was the high drop-out rate in the intervention group. However, under the assumption of most missing data being missing at random, linear mixed models still yield unbiased effect estimates ${ }^{(50)}$. Nevertheless, future research should focus on ways to keep frail or diseased participants in a study, for example by optimising the usability of interventions.

In conclusion, the findings of this study suggest that an eHealth intervention for nutrition screening and education can lead to improved nutritional status in older adults at risk of undernutrition and that it can lead to improved adherence to guidelines for a healthy diet and physical activity in community-dwelling older adults. More insight is needed into how such interventions yield more impact, for example by studying the intervention's delivery, acceptability and applicability in more detail, and by unravelling the intervention's mechanism of impact.

\section{Acknowledgements}

The authors would like to thank all participants, involved health care professionals, and the boards of Zorggroep NoordwestVeluwe and Opella. Furthermore, the authors would like to thank Mirthe Groothuis for her contribution to the coordination and data collection of the study, Jan Harryvan for his technical support, Hendriek Boshuizen for her advice about the statistical analyses, and Teuni Rooijackers, Nicolet Antonissen, Britt Hoens, Esther Lokerse and Dorien Oostra for their contribution to the recruitment of participants and data collection of the study.

This work was supported by the European Union, grant no. CIP-ICT-PSP-2013-7. The European Union had no role in the design, analysis or writing of this article.

M. N. v. D.-v. A. coordinated implementation of the study, analysed the data and drafted the article. All other authors critically read and revised the manuscript. M. N. v. D.-v. A., J. H. M. d. V., A. H.-N., L. C. P. G. M. d. G., M. M. v. B. and M. F. participated in the study design. M. F. contributed to the implementation of the intervention.

None of the authors has any conflicts of interest to declare.

\section{References}

1. Stratton RJ, Green CJ \& Elia M (2003) Disease-Related Malnutrition: An Evidence-Based Approach to Treatment. Wallingford: CABI Publishing. 
2. Rasheed S \& Woods RT (2013) Malnutrition and quality of life in older people: a systematic review and meta-analysis. Ageing Res Rev 12, 561-566.

3. Hickson M (2006) Malnutrition and ageing. Postgrad Med J 82, $2-8$

4. Kaiser MJ, Bauer JM, Ramsch C, et al. (2010) Frequency of malnutrition in older adults: a multinational perspective using the mini nutritional assessment. J Am Geriatr Soc 58, $1734-1738$

5. CBS (2017) Bevolking; kerncijfers (Population; key figures). http://bit.ly/2hp7TBP (accessed September 2017).

6. Milne AC, Potter J, Vivanti A, et al. (2009) Protein and energy supplementation in elderly people at risk from malnutrition. Cochrane Database Syst Rev, issue 2, CD003288.

7. Miller SL \& Wolfe RR (2008) The danger of weight loss in the elderly. J Nutr Health Aging 12, 487-491.

8. Elia M, Zellipour L \& Stratton RJ (2005) To screen or not to screen for adult malnutrition? Clin Nutr 24, 867-884

9. Halfens RJG, Meesterberends E, Neyens JCL, et al. (2016) Landelijke Prevalentiemeting Zorgproblemen. Rapportage resultaten 2015. Maastricht: CAPHRI School for Public Health and Primary Care.

10. Ziylan C, Haveman-Nies A, van Dongen EJI, et al. (2015) Dutch nutrition and care professionals' experiences with undernutrition awareness, monitoring, and treatment among community-dwelling older adults: a qualitative study. $B M C$ Nutr 1, 38.

11. van der Pols-Vijlbrief R, Wijnhoven HA, Schaap LA, et al. (2014) Determinants of protein-energy malnutrition in community-dwelling older adults: a systematic review of observational studies. Ageing Res Rev 18, 112-131.

12. Donini LM, Scardella P, Piombo L, et al. (2013) Malnutrition in elderly: social and economic determinants. J Nutr Health Aging 17, 9-15.

13. Lyons BP (2014) Nutrition education intervention with community-dwelling older adults: research challenges and opportunities. J Community Health 39, 810-818.

14. Sahyoun NR, Pratt CA \& Anderson A (2004) Evaluation of nutrition education interventions for older adults: a proposed framework. I Am Diet Assoc 104, 58-69.

15. Eysenbach G (2001) What is e-health? J Med Internet Res 3, E20.

16. Ludwig W, Wolf KH, Duwenkamp C, et al. (2012) Healthenabling technologies for the elderly-an overview of services based on a literature review. Comput Methods Programs Biomed 106, 70-78

17. Kaiser MJ, Bauer JM, Ramsch C, et al. (2009) Validation of the Mini Nutritional Assessment short-form (MNA-SF): a practical tool for identification of nutritional status. J Nutr Health Aging 13, 782-788.

18. Wilson MM, Thomas DR, Rubenstein LZ, et al. (2005) Appetite assessment: simple appetite questionnaire predicts weight loss in community-dwelling adults and nursing home residents. Am J Clin Nutr 82, 1074-1081.

19. van Lee L, Feskens EJ, Meijboom S, et al. (2016) Evaluation of a screener to assess diet quality in the Netherlands. Br J Nutr 115, 517-526.

20. van Doorn-van Atten MN, Haveman-Nies A, Pilichowski P, et al. (2018) Telemonitoring to improve nutritional status in community-dwelling elderly: design and methods for process and effect evaluation (In the Press)

21. Nykänen I, Rissanen TH, Sulkava R, et al. (2014) Effects of individual dietary counseling as part of a Comprehensive Geriatric Assessment (CGA) on nutritional status: a population-based intervention study. J Nutr Health Aging 18, $54-58$.
22. Ahn JA, Park J \& Kim CJ (2017) Effects of an individualised nutritional education and support programme on dietary habits, nutritional knowledge and nutritional status of older adults living alone. J Clin Nurs (epublication ahead of print version 7 September 2017).

23. Hoekstra JC, Goosen JH, de Wolf GS, et al. (2011) Effectiveness of multidisciplinary nutritional care on nutritional intake, nutritional status and quality of life in patients with hip fractures: a controlled prospective cohort study. Clin Nutr $\mathbf{3 0}$, 455-461.

24. Bandayrel K \& Wong S (2011) Systematic literature review of randomized control trials assessing the effectiveness of nutrition interventions in community-dwelling older adults. $J$ Nutr Educ Behav 43, 251-262.

25. Lutomski JE, Baars MAE, Schalk BWM, et al. (2013) The Development of the Older Persons and Informal Caregivers Survey Minimum DataSet (TOPICS-MDS): a large-scale data sharing initiative. PLOS ONE 8, e81673.

26. Folstein MF, Folstein SE \& McHugh PR (1975) 'Mini-mental state'. A practical method for grading the cognitive state of patients for the clinician. J Psychiatr Res 12, 189-198.

27. Vellas B, Villars H, Abellan G, et al. (2006) Overview of the MNA - its history and challenges. J Nutr Health Aging 10, 456-463; discussion 463-455.

28. Gezondheidsraad (2006) Richtlijnen goede voeding 2006 (Guidelines for Good Nutrition 2006). Den Haag: Gezondheidsraad.

29. Nordic Council of Ministers (2014) Nordic Nutrition Recommendations 2012: Integrating Nutrition and Physical Activity. Copenhagen: Nordic Council of Ministers.

30. Ocke M, Buurma-Rethans E, de Boer E, et al. (2013) Diet of Community-dwelling Older Adults: Dutch National Food Consumption Survey Older Adults 2010-2012. Bilthoven: Rijksinstituut voor Volksgezondheid en Milieu RIVM.

31. Laan W, Zuithoff NP, Drubbel I, et al. (2014) Validity and reliability of the Katz-15 scale to measure unfavorable health outcomes in community-dwelling older people. J Nutr Health Aging 18, 848-854.

32. Guralnik JM, Simonsick EM, Ferrucci L, et al. (1994) A short physical performance battery assessing lower extremity function: association with self-reported disability and prediction of mortality and nursing home admission. J Gerontol 49 , M85-M94.

33. Ware JE Jr. \& Sherbourne CD (1992) The MOS 36-item shortform health survey (SF-36). I. Conceptual framework and item selection. Med Care 30, 473-483.

34. Aaronson NK, Muller M, Cohen PD, et al. (1998) Translation, validation, and norming of the Dutch language version of the SF-36 Health Survey in community and chronic disease populations. J Clin Epidemiol 51, 1055-1068.

35. Nijs KA, de Graaf C, Siebelink E, et al. (2006) Effect of family-style meals on energy intake and risk of malnutrition in Dutch nursing home residents: a randomized controlled trial. J Gerontol A Biol Sci Med Sci 61, 935-942.

36. Southgate KM, Keller HH \& Reimer HD (2010) Determining knowledge and behaviour change: after nutrition screening among older adults. Can J Diet Pract Res 71, 128-133.

37. Benedict JA, Wilson D, Snow G, et al. (2000) Use of nutrition screening to develop and target nutrition education interventions for Nevada's elderly. J Nutr Elderly 19, 31-47.

38. Klein GL, Kita K, Fish J, et al. (1997) Nutrition and health for older persons in rural America: a managed care model. $J \mathrm{Am}$ Diet Assoc 97, 885-888.

39. Leggo M, Banks $\mathrm{M}$, Isenring $\mathrm{E}$, et al. (2008) A quality improvement nutrition screening and intervention program available to Home and Community Care eligible clients. Nutr Diet 65, 162-167. 
40. Keller HH, Haresign H \& Brockest B (2007) Process evaluation of bringing nutrition screening to seniors in Canada (BNSS). Can J Diet Pract Res 68, 86-91.

41. Wunderlich S, Bai Y \& Piemonte J (2011) Nutrition risk factors among home delivered and congregate meal participants: need for enhancement of nutrition education and counseling among home delivered meal participants. J Nutr Health Aging 15, 768-773.

42. Hamirudin $\mathrm{AH}$, Charlton $\mathrm{K}$ \& Walton K (2016) Outcomes related to nutrition screening in community living older adults: a systematic literature review. Arch Gerontol Geriatr 2016, 9-25.

43. Lara J, O'Brien N, Godfrey A, et al. (2016) Pilot randomised controlled trial of a web-based intervention to promote healthy eating, physical activity and meaningful social connections compared with usual care control in people of retirement age recruited from workplaces. PLOS ONE 11, e0159703.

44. Bernstein A, Nelson ME, Tucker KL, et al. (2002) A homebased nutrition intervention to increase consumption of fruits, vegetables, and calcium-rich foods in community dwelling elders. J Am Diet Assoc 102, 1421-1427.
45. Celis-Morales C, Lara J \& Mathers JC (2015) Personalising nutritional guidance for more effective behaviour change. Proc Nutr Soc 74, 130-138.

46. Bravata DM, Smith-Spangler C, Sundaram V, et al. (2007) Using pedometers to increase physical activity and improve health: a systematic review. JAMA 298, 2296-2304.

47. Snyder A, Colvin B \& Gammack JK (2011) Pedometer use increases daily steps and functional status in older adults. $J \mathrm{Am}$ Med Dir Assoc 12, 590-594

48. Harris T, Kerry SM, Victor CR, et al. (2015) A primary care nurse-delivered walking intervention in older adults: PACE (pedometer accelerometer consultation evaluation)Lift cluster randomised controlled trial. PLOS Med 12, e1001783.

49. van den Berg N, Schumann M, Kraft K, et al. (2012) Telemedicine and telecare for older patients - a systematic review. Maturitas 73, 94-114.

50. Bell ML, Kenward MG, Fairclough DL, et al. (2013) Differential dropout and bias in randomised controlled trials: when it matters and when it may not. BMJ 346, e8668. 